\title{
Gelation and Yielding Behavior of Polymer-Nanoparticle Hydrogels
}

\author{
Abigail K. Grosskopf ${ }^{1}$, Olivia A. Saouaf ${ }^{2}$, Hector Lopez Hernandez ${ }^{2}$, and Eric \\ A. Appel $2,3,4,5,{ }^{*}$ \\ ${ }^{1}$ Department of Chemical Engineering, Stanford University, Stanford, CA, 94305, USA \\ ${ }^{2}$ Department of Materials Science and Engineering, Stanford University, Stanford, CA, 94305, USA \\ ${ }^{3}$ Department of Pediatrics - Endocrinology, Stanford University, Stanford, CA, 94305, USA \\ ${ }^{4}$ Department of Bioengineering, Stanford University, Stanford, CA, 94305, USA \\ ${ }^{5}$ ChEM-H Institute, Stanford University, Stanford, CA, 94305, USA \\ *Correspondence: eappel@stanford.edu
}

\begin{abstract}
Polymer-Nanoparticle hydrogels are a unique class of self-assembled, shear-thinning, yield-stress fluids which have demonstrated potential utility in many impactful applications. Here we present a thorough analysis of the gelation and yielding behavior of these materials with respect to the polymer and nanoparticle component stoichiometry. Through comprehensive rheological and diffusion studies, we reveal insights into the structural dynamics of the polymer nanoparticle network which identify that stoichiometry plays a key role in gelation and yielding, ultimately enabling the development of hydrogel formulations with unique shear-thinning and yield-stress behaviors. Access to these materials opens new doors for interesting applications in a variety of fields including tissue engineering, drug delivery, and controlled solution viscosity.
\end{abstract}

\section{Introduction}

Shear-thinning hydrogels are unique and promising tools for controlling the delivery of therapeutics and cells, controlling solution viscosity, and 3D printing ${ }^{1}$. Polymer-Nanoparticle (PNP) hydrogels are a class of self-assembled hydrogels generated from dynamic, multivalent, and entropically-driven non-covalent interactions between polymeric nanoparticles and high-molecular-weight polymers ${ }^{2}$. These materials have been utilized for many applications ranging from prolonged delivery of therapeutic molecules and cells, easily applied and highly effective post-operative adhesion barriers, stabilization of biopharmaceuticals to improve cold-chain resilience, bio-inks for 3D printing, and prolonged delivery of wildland fire retardants for wildfire prevention ${ }^{3-12}$. Several important characteristics underlie why these hydrogels have demonstrated such broad utility: (i) ease of fabrication and scalability, (ii) high degree of shear-thinning enabling facile administration by injection or spraying, (iii) rapid recovery of mechanical properties following shearing, and (iv) tunable yield stress behavior enabling them to form robust depots or coatings after application ${ }^{13,14}$. Additionally, when formulated at relatively high weight percent of solids (typically up to $12 \mathrm{wt} \%$ ), these materials exhibit a small effective mesh size and thus very slow diffusion of embedded cargo compared to many other commonly used self-assembled hydrogels (e.g., alginate) ${ }^{15-17}$. While recent research has revealed many of the driving factors for the dynamic mechanical properties and temperature responsiveness of materials comprising mixtures of interacting polymers and nanoparticles, many of which have focused on polymeric melt systems, the exact interaction mechanisms which dominate gelation (e.g., bridging of polymers between particles or jamming of polymer-coated particles) under various formulation conditions are still poorly understood ${ }^{18-20}$. For this reason, we sought to design and execute a series of rheology and diffusion studies to elucidate the dominant mechanisms occurring in gelation of the PNP hydrogel system.

While basic mechanical studies have been performed on these complex PNP hydrogel materials in contexts relevant for various applications, a detailed and systematic investigation on the impact of the loading of each of polymer and nanoparticle components on the resulting PNP hydrogel properties has not yet been been performed. Moreoever, while many rheological studies on model hydrogel systems have been performed in the past to understand mechanistic behavior, rarely are thorough mechanistic studies performed on promising novel materials which tend 
to be highly structurally complex and multifaceted in their mechanical behaviors. Indeed, many studies on model materials focus on evaluating a singular material characteristic of interest, rather than the multifaceted group of material properties relevant for engineering applications. By titrating the amounts of polymer and nanoparticle in the PNP hydrogel formulation independently, it is possible to gain insight on the linear viscoelastic, yielding, and flow behavior simultaneously to further elucidate which components lead to the unique and useful properties exhibited by the PNP hydrogel system. Herein we use shear rheology and diffusion studies on a wide range of PNP hydrogel formulations to reveal the compositional features yielding unique and desirable properties in these materials.

\section{Results}

\section{Formulation of PNP Hydrogels}

PNP hydrogels are formulated by simply mixing poly(ethylene glycol)-b-poly(lactic acid) nanoparticles (PEG-PLA NPs) and dodecyl-modified hydroxypropylmethylcellulose polymers (HPMC- $\mathrm{C}_{12}$; Figure 1a, Supplementary Figures 1-3). PEG-PLA NPs are easily scaled, biodegradable and have been used clinically in the past ${ }^{21,22}$. HPMC is ubiquitously used as an excipient and is easily modified ${ }^{23}$. The polymers are theorized to form a dynamic corona around the nanoparticles bridging between nanoparticles ${ }^{2,18}$. Previous studies of PNP hydrogel systems have demonstrated that increasing the hydrophobicity of the HPMC modification, either by increasing the size of the hydrophobic moieties used for the modification (e.g., $\mathrm{C}_{12}$ vs. $\mathrm{C}_{6}$ ) or by increasing the total amount of hydrophobic modification along the chains, leads to stronger PNP interactions and increased hydrogel viscoelasticity ${ }^{18}$, suggesting that hydrophobic interactions play a larger role in gel formation than hydrogen bonding between the HPMC and PEG polymers. Herein we utilized a consistent level of dodecyl modification and concentrated our efforts on understanding the effects of P-NP stoichiometry on gelation, viscoelasticity, and flow properties. The trends observed in these studies are expected to still apply generally to PNP hydrogels if either the degree of HPMC modification or the identify of the hydrophobic moieties is adjusted.

a

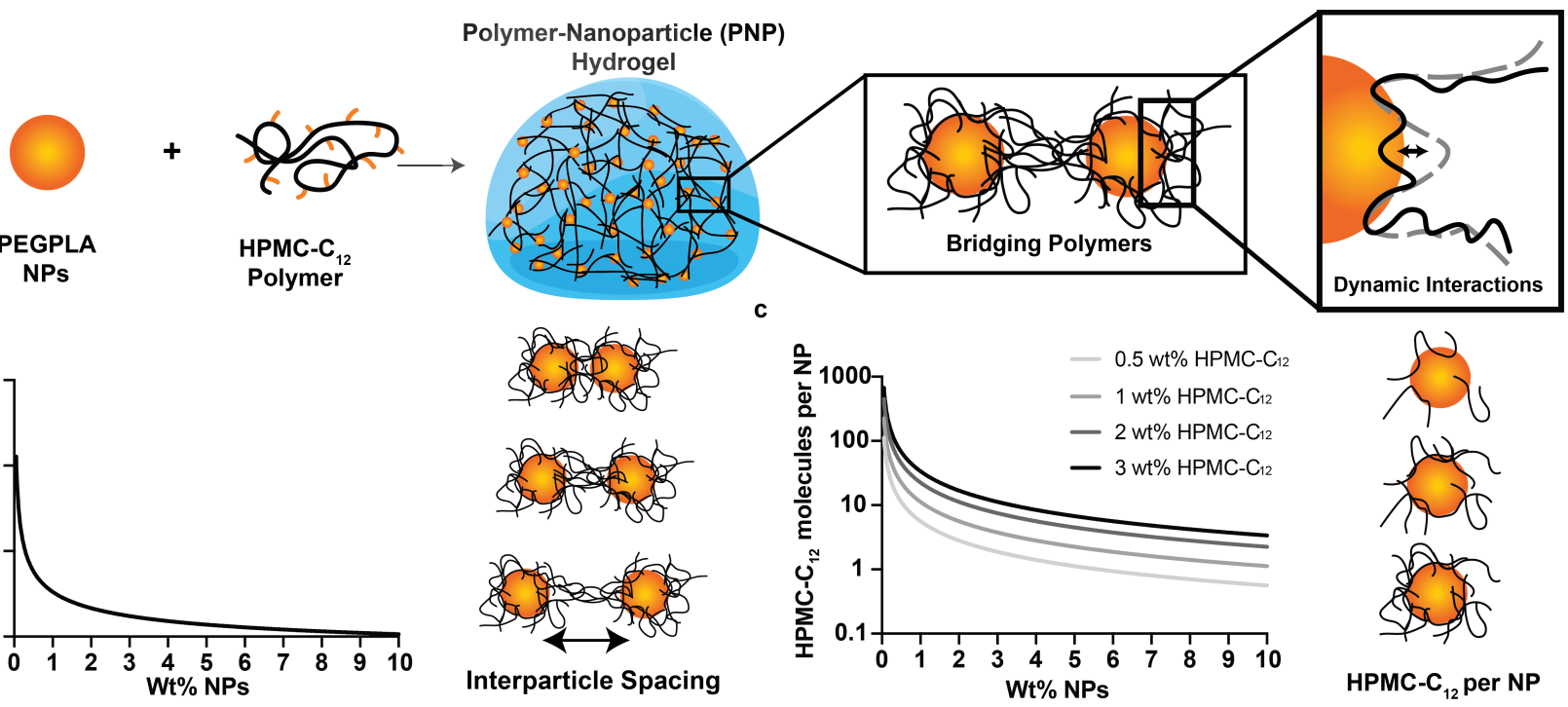

Figure 1. a, PNP Hydrogels form through the interactions of PEG-PLA nanoparticles (NPs) and dodecyl-modified hydroxypropyl methylcellulose polymers $\left(\mathrm{HPMC}-\mathrm{C}_{12}\right)$. Polymers bridge between polymers and dynamically interact with the NP surface. $\mathbf{b}$, Interparticle spacing of NPs as a function of the weight percent of NPs added. c Number of molecules of HPMC-C 12 per NP as a function of the concentration of NPs and concentration of polymer.

Calculations reveal that the average interparticle spacing of nanoparticles greatly decreases with the amount of nanoparticles added to the hydrogel (Figure 1b). Indeed, at $10 \mathrm{wt} \% \mathrm{NPs}$, the nanoparticles are nearly touching. Additionally, calculations reveal that the number of HPMC- $\mathrm{C}_{12}$ polymer molecules per nanoparticle is greatly dependent on the polymer concentration (Figure 1C). Formulations are referred to in the format P-NP, whereby $P$ refers to the weight percent of HPMC- $\mathrm{C}_{12}$ and NP refers to the weight percent of the PEG-PLA NPs (n.b., the remaining mass of the formulation is phosphate-buffered saline). 


\section{Network Diffusion}

To gain greater insight into the structural dynamics of the components within the PNP hydrogels, fluorescence recovery after photobleaching (FRAP) microscopy experiments were performed to assess the diffusion of the polymer and nanoparticle components independently (Figure 2). The HPMC- $\mathrm{C}_{12}$ and PEG-PLA NP components were first labelled with fluorescent probes to enable this analysis ${ }^{17}$. A diffusion coefficient was calculated based on recovery data obtained from the FRAP experiments ${ }^{4,24}$. Data were collected until a plateau was reached or background photobleaching began to interfere (typically 30 minutes of data collection per FRAP experiment). An immobile fraction was calculated based on the signal that was not recovered once a plateau was reached.

We first conducted FRAP experiments with fluorescently-labelled PEG-PLA NPs (Figure 2). A control solution of $5 \mathrm{wt} \%$ nanoparticles and no HPMC- $\mathrm{C}_{12}$ polymer exhibited rapid diffusion at a rate of approximately $3.5 \mathrm{um}^{2} / \mathrm{s}$. In contrast, the PEG-PLA NPs in hydrogels exhibited almost no diffusion and surprisingly high immobile fractions such that meaningful diffusion analysis was not feasible. These results suggest that even a very small amount of polymer crosslinks the NPs such that they become arrested in the hydrogel network, greatly reducing NP diffusion.
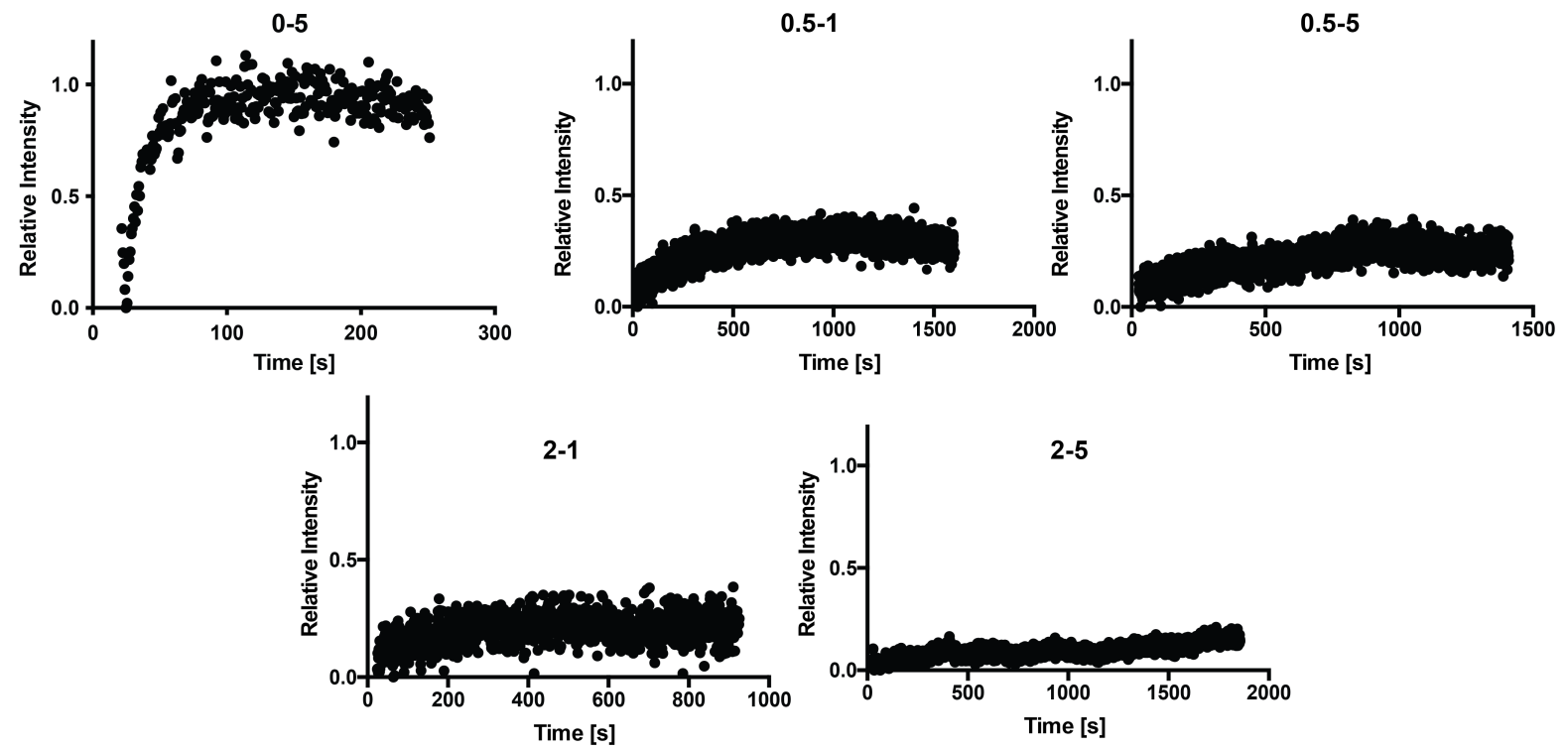

Figure 2. Fluorescence recovery after photobleaching (FRAP) experiments of fluorescently-labelled PEG-PLA NPS were performed to evaluate NP diffusion. Recovery data is normalized by the original maximum signal and minimum signal at bleaching. Formulation notation denotes P-NP weight percent loadings in the PNP hydrogels (n.b., the remaining mass of the formulation is phosphate-buffered saline). Representative data for each PNP formulation is shown.

We then sought to evaluate the diffusion of the HPMC- $\mathrm{C}_{12}$ component within the PNP hydrogels (Figure 3). While polymer diffusion varied slightly with formulation, the data demonstrate that formulations with greater solid content, and thus higher viscosity, exhibited decreased polymer diffusion. Significant immobile fractions were observed in many formulations, though a comparison of the diffusion of the polymer in PNP hydrogels with a control polymer solution provides important mechanistic insight. For example, the 0.5-1 formulation exhibited much lower polymer diffusion compared to the the $0.5-0$ control solution, indicating that addition of only $1 \mathrm{wt} \%$ particles had a profound impact on polymer diffusion. In contrast, the 2-1 formulation did not have such a reduced polymer diffusion compared to the 2-0 control solution. Interestingly, even though the 2-5 formulation clearly exhibited a much higher viscosity by eye prior to running the FRAP experiments, the polymer diffusion in the $0.5-5$ formulation was determined to be lower than in the 2-5 formulation.

\section{PNP Hydrogel Gelation}

To investigate PNP hydrogel viscoelasticity, the shear rheology of formulations independently titrating the nanoparticle and polymer content was evaluated on a serrated parallel plate rheometer (Figure 4). Frequency sweeps were performed to investigate viscoelasticity across multiple timescales. Increasing nanoparticle content at a constant polymer content led to a more solid-like rheological response. As nanoparticle content increases, the materials become stiffer and more solid-like, exhibiting a higher G' and lower $\tan (\delta)$ (G'/G'), across a broad range 

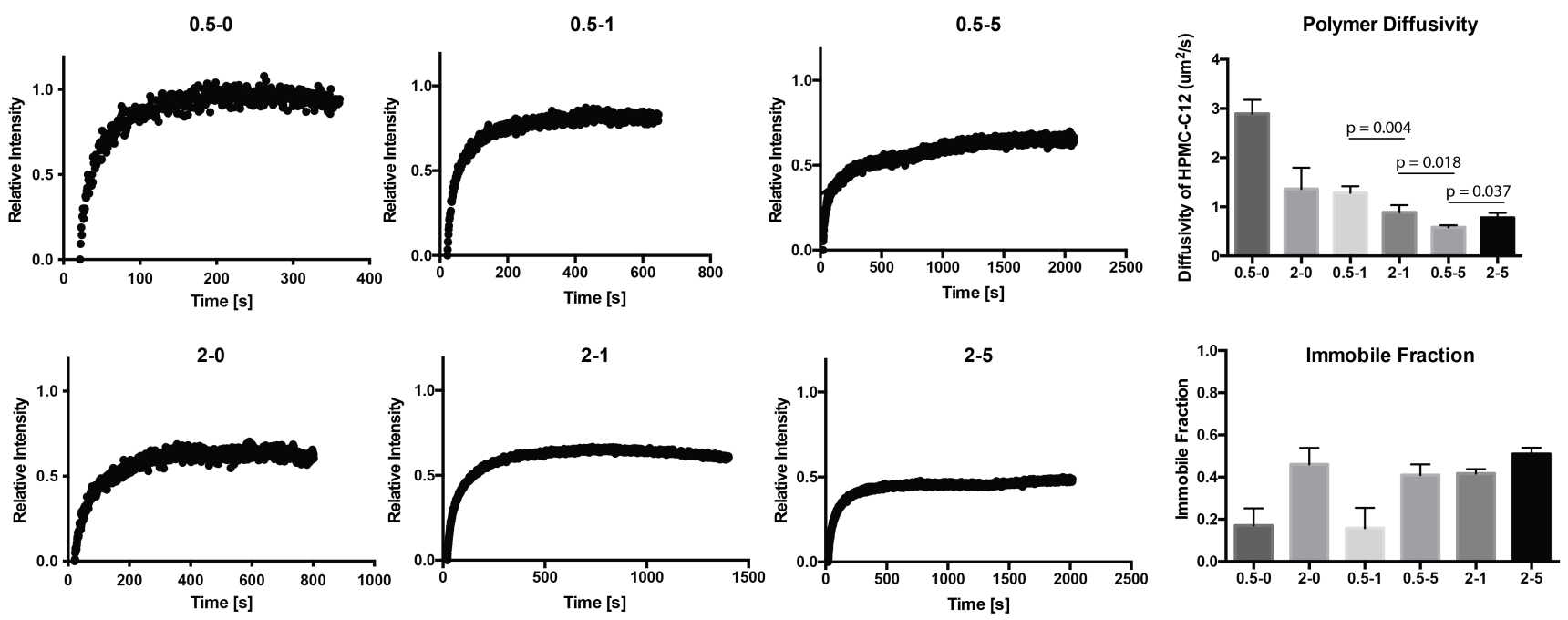

Figure 3. Fluorescence recovery after photobleaching (FRAP) of fluorescently-labelled HPMC- $_{12}$ to understand diffusion with representative recovery curves for each PNP formulation evaluated. Recovery data is normalized by the original maximum signal and minimum signal at bleaching. The diffusivity from recovery analysis of multiple samples $(n=3-4)$ and corresponding immobile fractions are presented. Formulations are referred to the polymer weight percent followed by the nanoparticle weight percent (with the rest of the weight percent being phosphate-buffered saline).

of frequencies. The more shallow slope of the G' suggests a more Rouse-like frequency-dependent rheological response ${ }^{25}$. The crossover point of the G' and G" values, representing a network relaxation time, shifts from right to left with an increase in nanoparticles (e.g., shorter timescales to longer timescales), with the crossovers of the 2-1 and 2-5 formulations no longer visible in the measurable frequency range. Cole-cole representations of these data are shown in Supplementary Figure 4, demonstrating that these materials do not follow Maxwell relaxation behavior, and that the addition of nanoparticles greatly alters relaxation timescales ${ }^{26}$.

In contrast, increasing the polymer content at a constant nanoparticle content gave a surprising result. PNP hydrogels with more polymer were generally stiffer, exhibiting a higher G', but also demonstrated a more liquid-like response with a higher $\tan (\delta)$ and a greater frequency-dependence of $G$ '. Moreover, in contrast to the effect observed for addition of nanoparticles, increasing the polymer led the G' and G" crossover to shift to the right, indicating that increased polymer leads to shorter relaxation times. Assessing all frequency sweeps together, the results suggest that formulations with excess polymer in relation to nanoparticles (i.e. higher P-NP ratio) exhibit more dissipative characteristics similar to a polymer solution or melt. More solid content in the hydrogel, therefore, does not necessarily yield more solid-like responses, but rather stoichiometry plays a crucial role in gelation. Similar observations have been made previously in the design of supramolecular hydrogels formed through metal-ligand interactions ${ }^{27}$.

\section{Yielding Response}

PNP hydrogels have shown great promise in use for biomedical research as injectable materials for controlled delivery of pharmaceuticals and cells. In order to be easily injected through standard syringes yet also form a robust depot in the body following administration, hydrogels must exhibit yield stress behavior. Additionally, injectable hydrogels, including PNP hydrogels, have been shown to reduce cell membrane damage during syringe needle injection by changing the flow velocity gradient within the syringe due to the significant shear-thinning and yield stress flow behavior ${ }^{12,28}$. Thus, yielding behavior of various formulations was investigated with amplitude sweeps of strain-dependent oscillatory rheology (Figure 5). At a constant polymer concentration, increasing the nanoparticle content led to greatly increased the strain-to-yield, which exceeded $500 \%$ strain for several formulations. Additionally, at a higher nanoparticle content an increase in the G" is observed during yielding, suggesting a significant deformation energy is lost by de-caging of the nanoparticles during the yielding response ${ }^{18,19,29}$. In contrast, increasing the polymer content at a constant nanoparticle content exhibited the opposite effect. Increased polymer content, particularly at high polymer:nanoparticle ratios, reduced the strain-to-yield. Indeed, formulations 


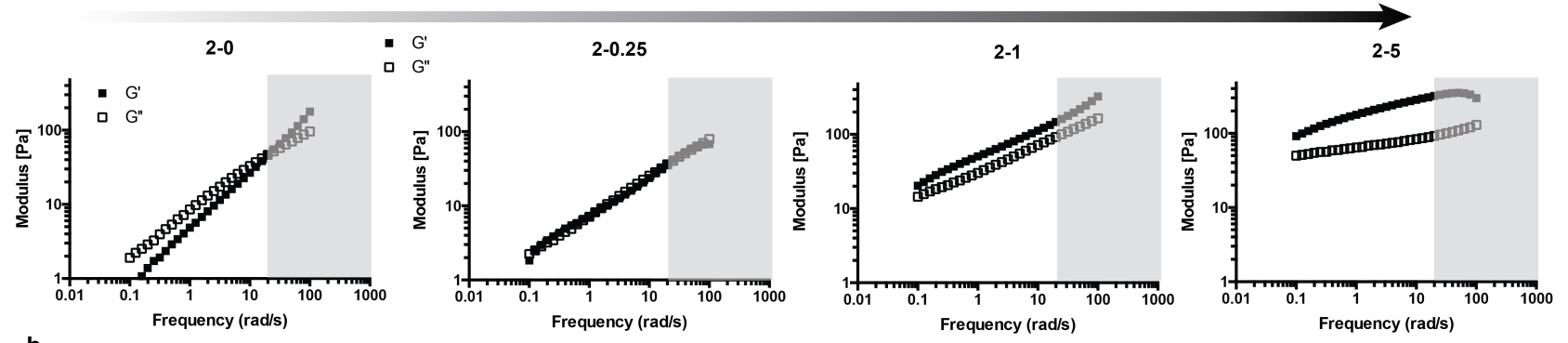

b

Increasing Polymer Content at a Constant Nanoparticle Content

0.5-1

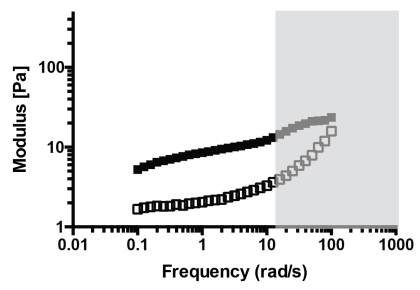

$1-1$

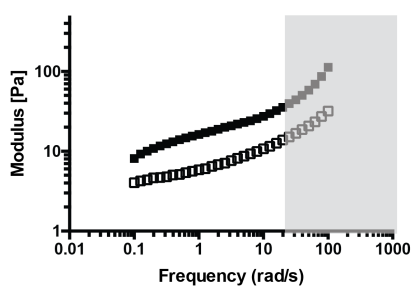

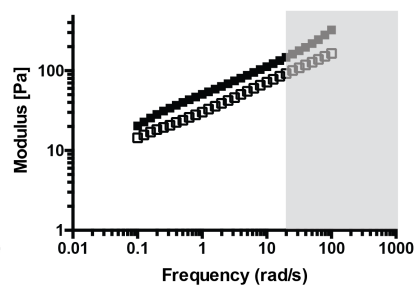

3-1

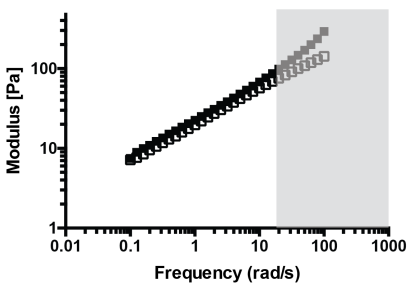

Figure 4. Oscillatory frequency sweeps of various PNP hydrogel formulations within the linear viscoelastic regime (1\% strain). Likely inertial artifacts are shaded in gray. a, Formulations with increasing nanoparticle content. b, Formulations with increasing polymer content. Formulations are referred to the polymer weight percent followed by the nanoparticle weight percent (with the rest of the weight percent being phosphate-buffered saline).

with lower polymer content exhibited increases in the G" during yielding while formulations higher polymer content did not. These results corroborate our findings described above that the stoichiometry of polymer to nanoparticle plays an important role in network dynamics.

Increasing Nanoparticle Content at a Constant Polymer Content

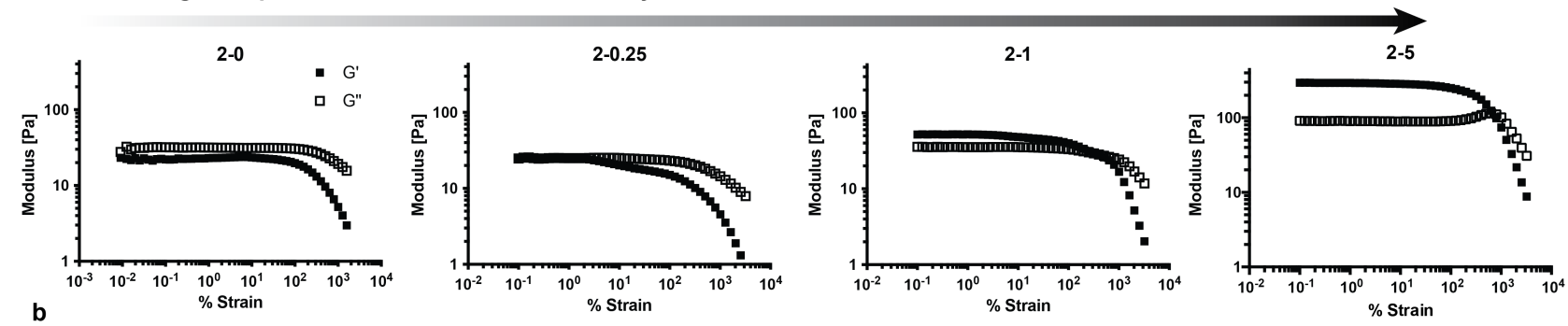

Increasing Polymer Content at a Constant Nanoparticle Content
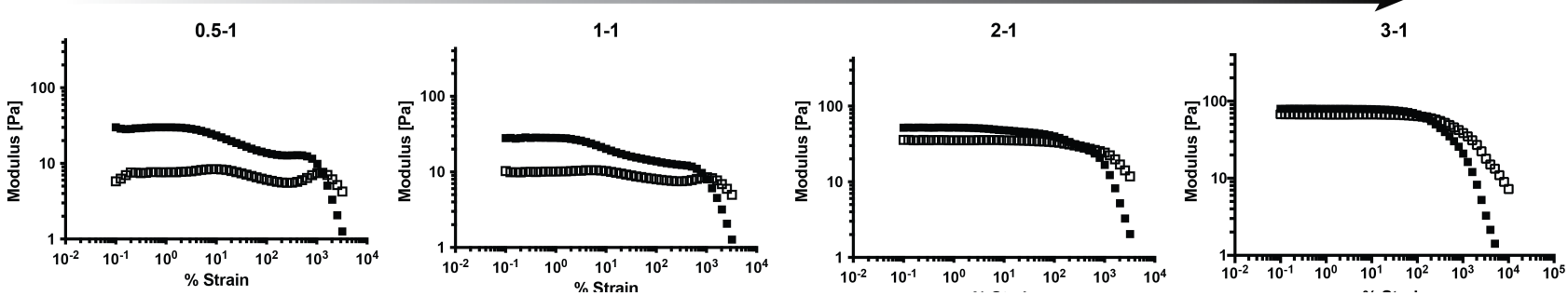

Figure 5. Oscillatory amplitude sweeps of various PNP hydrogel formulations at $10 \mathrm{rad} / \mathrm{s}$. a, Formulations with increasing nanoparticle content. b, Formulations with increasing polymer content. Formulations are referred to the polymer weight percent followed by the nanoparticle weight percent (with the rest of the weight percent being phosphate-buffered saline).

In addition to examining strain, steady shear flow sweeps varying the shear rate from high to low shear rate were performed to examine the yield stress behavior of the materials (Figure 6). Measuring yield stress behavior in 
complex soft materials is notoriously complex ${ }^{30}$. These materials exhibit unique flow-to-yield transitions that are best identified through visualization of the plots of the viscosity vs. stress and stress vs. shear rate. Upon yielding from high to low shear rate, there is a clear change in slope of the viscosity from a steep slope, characteristic of a shear-thinning material, to a more flat slope, characteristic of a yielded material (at low enough stress and shear rate). In the yielded regime, the measured viscosity in the flow sweep is an artifact (viscosities above $1000 \mathrm{~Pa}$ are unbelievably highly and are almost certainly artifacts) ${ }^{31}$. To identify the apparent yield stress from the flow sweep, we first used tangent lines on the stress and viscosity to determine the flow regime and pre-yield regime, and then fit the Herschel-Bulkley model to the shear-rate dependent stress data only in the flow regime ${ }^{32,33}$.

a

Increasing Nanoparticle Content at a Constant Polymer Content
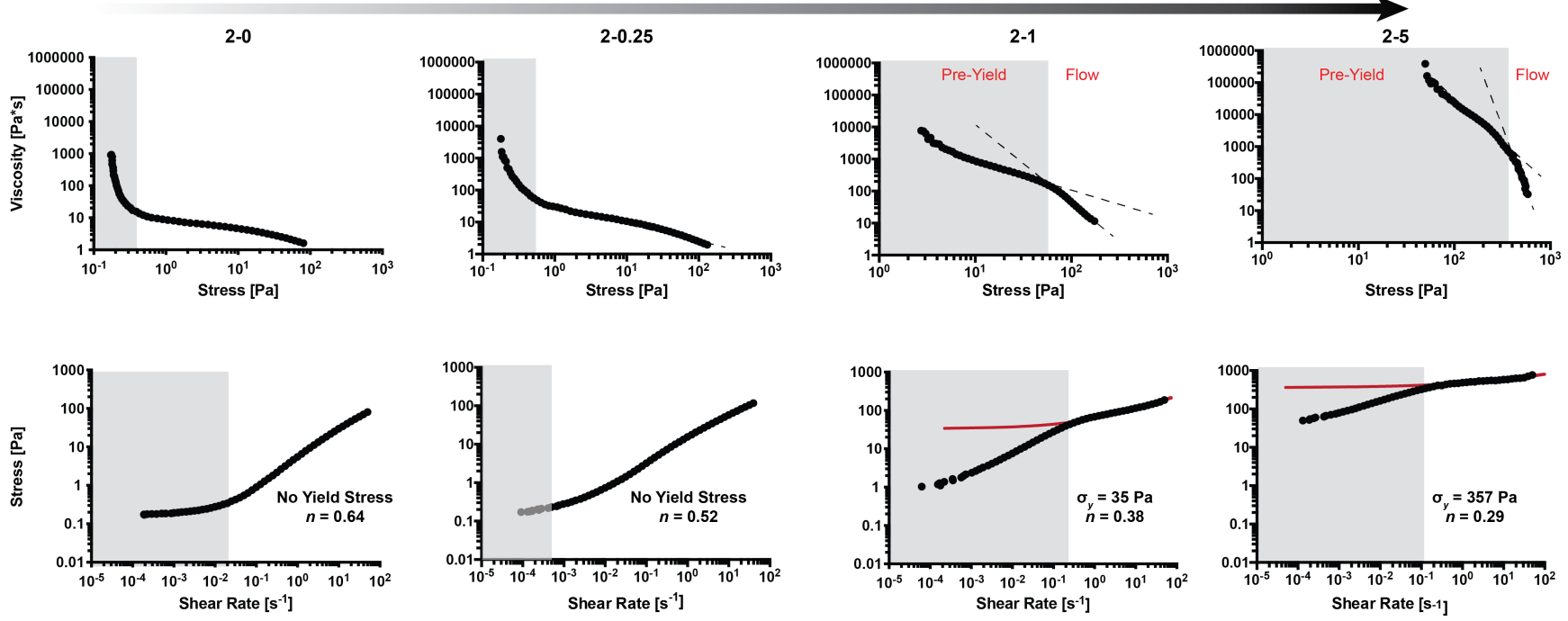

b

Increasing Polymer Content at a Constant Nanoparticle Content
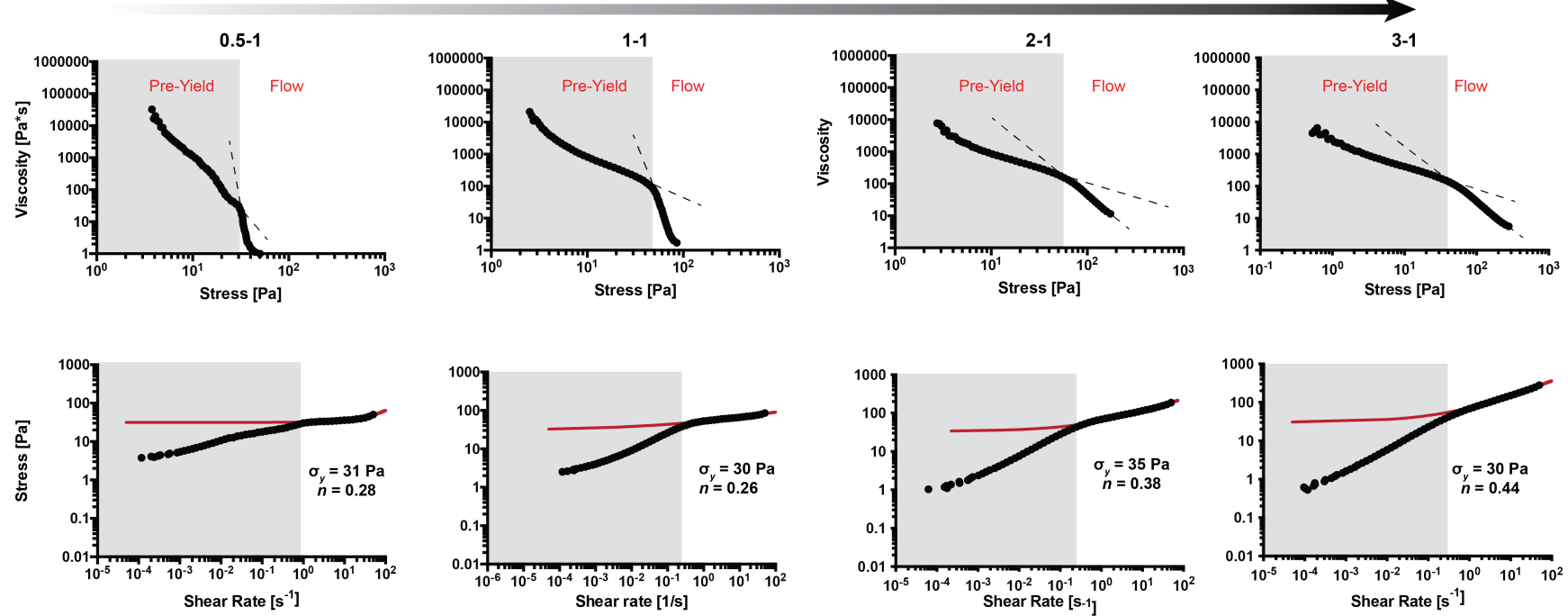

Figure 6. Flow sweeps of various PNP hydrogel formulations. The viscosity vs. stress (top) is plotted in addition to the corresponding stress vs. shear rate (bottom) for each formulation evaluated. Data that are artifact is shaded is grey. When a yield stress was observed, the pre-yield and flow regimes are labelled. On the Stress vs. shear rate sweeps, yield stress values $\left(\sigma_{y}\right)$ and consistency indices $(n)$ from Herschel-Bulkley fits are recorded. a, Formulations with increasing nanoparticle content. b, Formulations with increasing polymer content. Formulations are referred to the polymer weight percent followed by the nanoparticle weight percent (with the rest of the weight percent being phosphate-buffered saline).

These studies indicate that increasing the nanoparticle content at a constant polymer content led to increased yield stresses. Indeed, measurable yield stress values were only found at nanoparticle concentrations above $0.25 \%$. An increase in nanoparticle content also led to a lower shear-thinning exponent from the Herschel-Bulkley fits, implying a 
greater degree of shear-thinning behavior that is crucial for injectability. Interestingly, increasing polymer content at a constant nanoparticle content led to a constant yield stress across formulations, suggesting that nanoparticle content alone dictates yield stress behavior. Notably, even very weak hydrogels (i.e., low solids content and low modulus gels such as 0.5-1) exhibited significant yield stress behavior. Furthermore, while increased polymer content appeared to also increase shear-thinning behavior, flow sweeps performed on a capillary viscometer indicated that viscosity at high shear rates is dominated by the polymer content (Supplementary Figure 5), commensurate with our previous observations ${ }^{14}$.

\section{Elucidating the Design Rules for PNP Hydrogel formation}

Thus far in our studies, all formulations with at least $1 \mathrm{wt} \%$ nanoparticle content have been shown to exhibit robust solid-like rheological responses. Given that in handling, nanoparticles solutions act liquid-like, we sought to establish the point where hydrogel formation truly occurs by assessing formulations with very little polymer content but high nanoparticle content (Figure 7). While a simple $5 \mathrm{wt} \%$ nanoparticle solution (i.e., 0 wt\% polymer content) exhibited some viscoelastic response, a crossover of the G' and G" was observed in the measurable frequency regime, yielding was observed at a very low strain, and the solution exhibited a very low yield stress. Upon addition of only $0.25 \mathrm{wt} \%$ polymer, the a solid-like hydrogel materials forms that exhited no observable crossover of G' and G" in frequency sweep as well as a robust yield stress. Increasing the polymer content only slightly above this level to $0.5 \%$ yielded materials with frequency-independent moduli, dramatically increased strain-to-yield, and increased yield stress values. Interestingly, the $0.5-5$ formulation showed a slight increase in G" during yielding, while the 0.25-5 formulation did not. The 0.5-5 formulation also exhibited a lower strain-to-yield than the $0.5-1$ formulation, indicating that a higher nanoparticle content and lower polymer-to-nanoparticle stoichiometry increases the strain-to-yield. Herschel-Bulkley analysis was not possible for the flow data obtained for these formulations due to the significant artifacts in the data, leaving too few points for an effective fit.

a
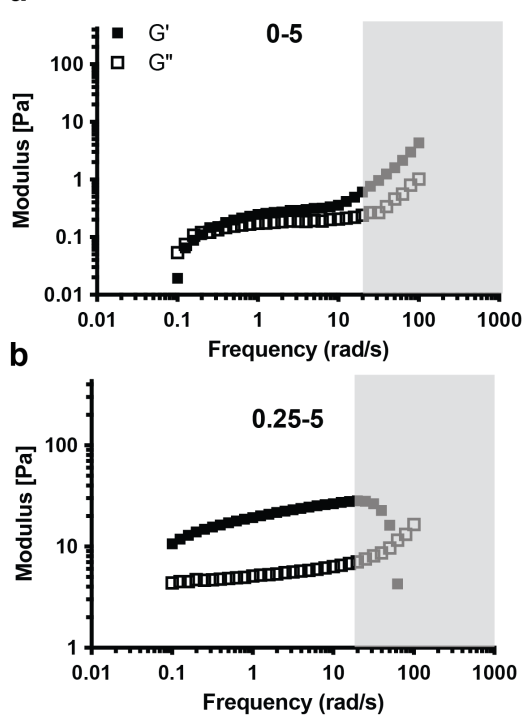

c

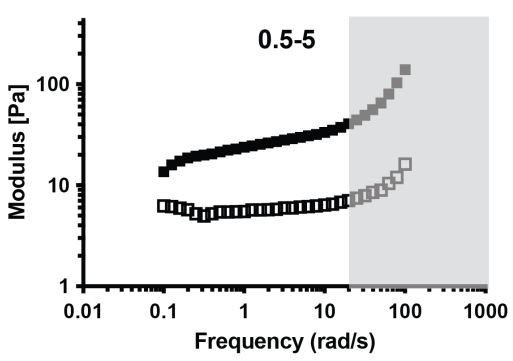

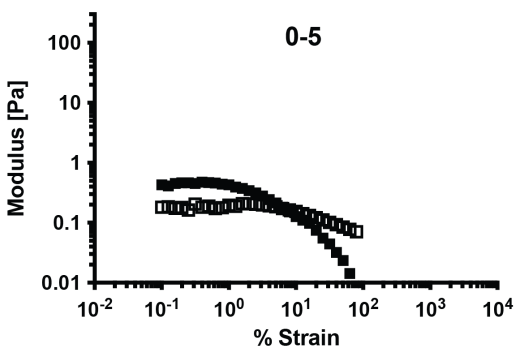
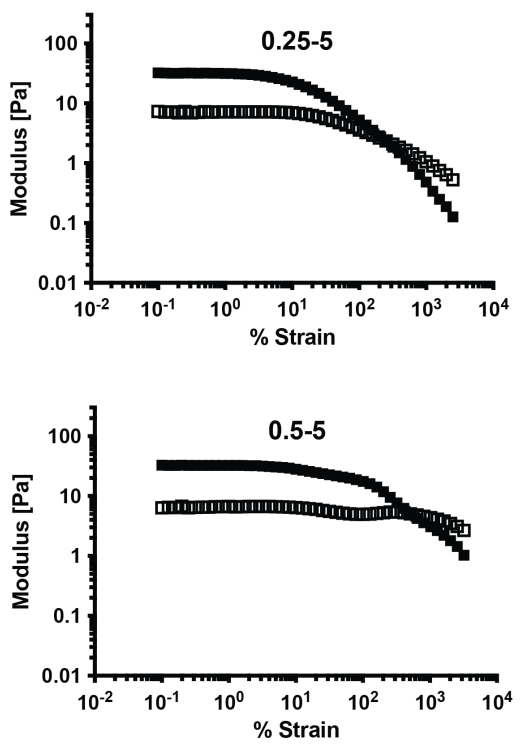
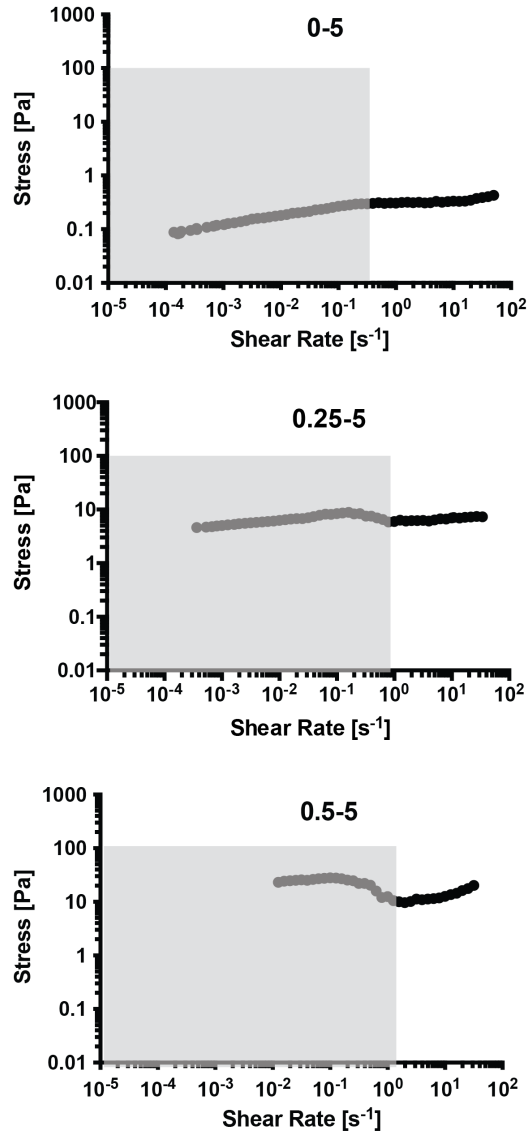

Figure 7. Rheological analysis of PNP formulations containing low polymer (a, $0-5, \mathbf{b}, 0.25-5, \mathbf{c} 0.5-5)$. Formulations are referred to the polymer weight percent followed by the nanoparticle weight percent (with the rest of the weight percent being phosphate-buffered saline). 


\section{Discussion}

Using a combination of studies of component diffusion and bulk rheology we have sought to elucidate the structure and gelation mechanisms underlying PNP hydrogels. FRAP provides information on the dynamics of the individual components of the system. Through FRAP diffusion studies we discovered that the PEG-PLA nanoparticles in the hydrogel are essentially arrested into a static network structure, while the HPMC- $\mathrm{C}_{12}$ polymers dynamically diffuse through this structure (Figure 8a). Increasing polymer content does not necessarily slow network dynamics, but rather too much excess polymer actually leads to "free" polymers that are not bound to nanoparticles. These free polymers therefore do not contribute to network formation and simply dissipate stress within the matrix.

a

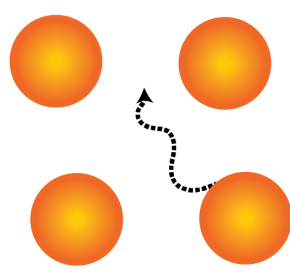

NPs Rapidly Diffuse
Polymer Bound and Bridge NP Excess Diffusing Polymer Enhancing Gelation

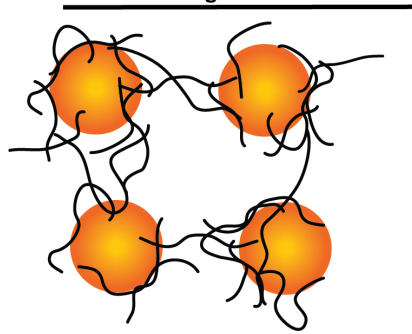

NPs Stabilized Lending Elasticity and Yield Stress

Dissipating the Matrix

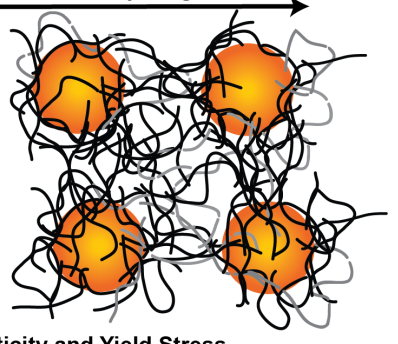

C

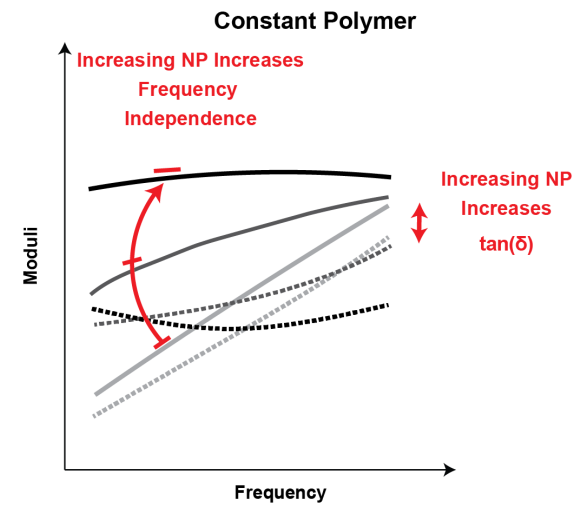

Figure 8. a, Schematic representation of the structural dynamics of the PNP hydrogel with increasing polymer. b, Schematic fictional data representations with annotation of the effect of nanoparticle increase with constant polymer content and polymer increase with constant nanoparticle content in the viscosity with increasing shear rate. c, Schematic fictional data representation with annotation of the effect of adding nanoparticle to constant polymer content.

A series of rheology studies corroborate these diffusion studies and demonstrate which components contribute to the unique mechanical characteristics of the PNP hydrogels. For example, the nanoparticles uniquely determine the yield stress (Figure 8b). Hydrogels with relatively higher NP content exhibit higher yield stresses, higher degrees of shear-thinning, and more frequency-independent solid-like hydrogel behavior in the linear viscoelastic regime (Figure 8c). In contrast, hydrogels with relatively higher polymer content are more viscous but exhibit a constant yield stress and a higher high-shear viscosity (i.e., relatively more challenging injectability). The mechanical properties for all of the formulations evaluated are summarized in Supplementary Figure 6 and can aid in formulating hydrogel materials to meet engineering specifications for any given application of interest. This study did not explore all possible formulations but simply provided design criteria, and stiffer PNP hydrogels can be formulated through the addition of a higher percentage of nanoparticles or a higher percentage of polymer. Furthermore, understanding the design principles behind these properties may enable us to create materials which push the boundaries of currently available hydrogels. One particularly interesting result from these studies is that it is possible to generate hydrogels with extremely low moduli and viscosities, yet high yield stress values ${ }^{34}$.

The results of these various studies imply that there is an optimal polymer-to-nanoparticle (P:NP) ratio lying between 0.1 and 1 in which there is synergy in the network leading to maximal solid-like hydrogel mechanics and yielding 
behavior (Figure 9). The most synergy appeared in the $0.5-1$ and 2-5 formulations, both formulations comprising $\mathrm{P}: \mathrm{NP}$ ratios close to 0.5 . Connecting our experimental results back to our calculations, we find that the P:NP ratio greater than 0.1 and less than 1 corresponds to a range of roughly 1 to $11 \mathrm{HPMC}-\mathrm{C}_{12}$ polymer chains per PEG-PLA nanoparticle with approximately 5 polymers per nanoparticle being most optimal. PNP hydrogel formation seems to be independent of the nanoparticle interparticle spacing, suggesting that jamming may play a negligible role in gelation and instead bridging of polymer chains between the nanoparticles is a more dominant mechanism of gelation. From a different perspective, however, yield stress is highly dependent on the nanoparticle content, which is likely a function of the interparticle spacing. This observation corroborates literature reports that imply percolation of colloids in colloidal gels leads to yield stress behavior ${ }^{35}$. Overall, thorough characterization of the PNP hydrogel platform has provided critical mechanistic insights underlying hydrogel formation and dictating hydrogel properties, enabling fine tuning of the mechanical properties for various applications of interest.
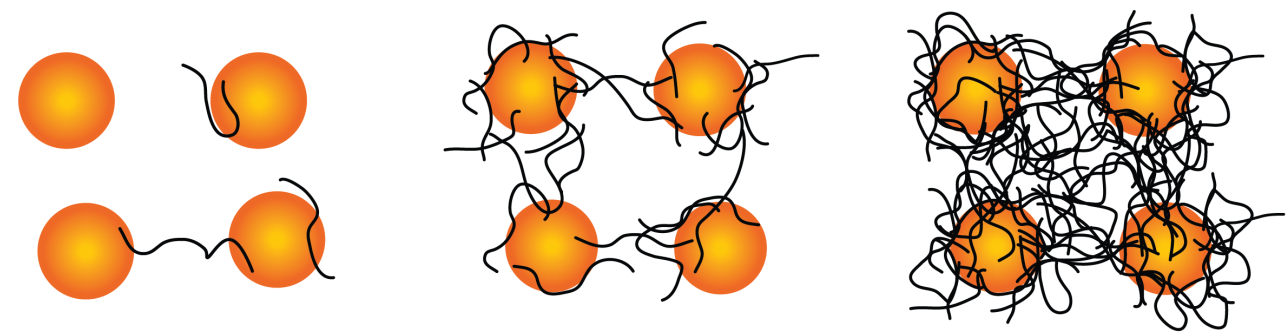

\begin{tabular}{c|c|c|c|} 
& $\begin{array}{c}\text { Nanoparticle-Dominated } \\
\text { P:NP Ratio }<0.1\end{array}$ & $\begin{array}{c}\text { Polymer-Nanoparticle Synergy } \\
0.1<\text { P:NP Ratio < }\end{array}$ & $\begin{array}{c}\text { Polymer-Dominated } \\
\text { P:NP Ratio }>1\end{array}$ \\
\cline { 2 - 4 } $\begin{array}{c}\text { Processing Window } \\
(\% \text { Strain At Yield) }\end{array}$ & $<500 \%$ & $>500 \%$ & $<500 \%$ \\
$\begin{array}{c}\text { Solid-like Behavior } \\
(\tan (\delta))\end{array}$ & $<0.5$ & $<0.5$ & $>0.5$ \\
$\begin{array}{c}\text { Frequency Dependence } \\
\left(\text { Slope of } G^{\prime} \sim \omega^{*}\right)\end{array}$ & $<0.25$ & $<0.25$ & $>0.25$ \\
$\begin{array}{c}\text { Yield Stress } \\
\text { (Relative to Solid-Content) }\end{array}$ & Medium & High & Low \\
$\begin{array}{c}\text { High Shear Viscosity } \\
\text { (Relative to Solid-Content) }\end{array}$ & Low & Medium & High
\end{tabular}

Figure 9. Stoichiometric regimes of PNP hydrogels based on the P:NP ratio and their resulting rheological characteristics relevant to engineering design.

\section{Conclusions}

Our study elucidates the design features underlying the gelation and yielding behavior of PNP hydrogels. The diffusion studies conducted here indicate that the nanoparticles become nearly completely arrested into a network while the polymer dynamically diffuses through the network. We find that increasing both polymer and nanoparticle yields stiffer materials, but that the P:NP ratio plays a large role in determining the solid-like properties of the resulting materials. Higher P:NP ratios (i.e., excess polymer present) leads to more liquid-like behavior, while low $P: N P$ ratios leads to lower energy networks. We find that a P:NP ratio between 0.1 and 1 leads to synergistic effects in the network, including dominant solid-like behavior and yielding behavior only at very high strains often in excess of $1000 \%$. Indeed, the most optimal P:NP ratio appeared to be approximately 0.5 . We additionally find that the nanoparticles play a key role in imparting a yield stress on the materials, while the polymer simply increases the viscosity at high shear rates, reducing shear-thinning behavior. Overall, these studies reveal that manipulation of the stoichiometry and total content of the polymer and nanoparticle components in PNP hydrogels provides a means to precisely tune the mechanical properties of these materials, enabling facile optimization for various applications of interest. 


\section{Methods}

\section{Materials}

All chemicals, reagents, and solvents were purchased as reagent grade from Sigma-Aldrich, Acros, or Alfa Aesar and used as received unless otherwise specified. Glassware and stir bars were oven-dried at $180{ }^{\circ} \mathrm{C}$. When specified, solvents were degassed by three cycles of freeze, pump, and thaw. HPMC-C ${ }_{12}$, and PEG-PLA were synthesized and characterized as described previously ${ }^{5}$. NPs were prepared by nanoprecipitation according to literature procedures, and NP size and dispersity were characterized by dynamic light scattering $\left(D_{\mathrm{H}} \sim 40 \mathrm{~nm}\right.$, PDI $<0.02)^{5}$.

\section{PEG-PLA Synthesis}

Procedure was followed and analyzed as described previously. ${ }^{5}$ PEG $(0.25 \mathrm{~g}, 4.1 \mathrm{mmol})$ and DBU $(10.6 \mathrm{mg}, 10 \mathrm{ml}$, $1.0 \mathrm{~mol} \%$ relative to LA) were dissolved in dichloromethane (DCM; $1.0 \mathrm{ml})$. LA $(1.0 \mathrm{~g}, 6.9 \mathrm{mmol})$ was dissolved in DCM $(3.5 \mathrm{ml})$ with mild heating. The LA solution was then added rapidly to the PEG/DBU solution and was allowed to stir rapidly for $10 \mathrm{~min}$. The PEG-PLA copolymer was then recovered from the reaction medium by precipitation from excess 50:50 mixture cold diethyl ether and hexanes, collected by filtration, and dried under vacuum to yield a white amorphous polymer. According to gel permeation chromatography (GPC), the resulting polymer exhibited: $\mathrm{Mn}(\Theta)=21 \mathrm{kDa}(1.08)$ (full GPC trace shown in Supplementary Figure 1). Mn, Mw, and dispersity values were determined via SEC implementing PEG standards (American Polymer Standards Corporation) after passing through two SEC columns (inner diameter, $7.8 \mathrm{~mm}$; Mw range 200 to 600,000 g mol1; Resolve Mixed Bed Low divinylbenzene (DVB) (Jordi Labs)] in a mobile phase of DMF with $0.1 \mathrm{M} \mathrm{LiBr}$ at $35^{\circ} \mathrm{C}$ and a flow rate of 1.0 $\mathrm{ml} / \mathrm{min}$ [Dionex UltiMate 3000 pump, degasser, and autosampler (Thermo Fisher Scientific)).

\section{HPMC- $\mathrm{C}_{12}$ Synthesis and Characterization}

Hypromellose (HPMC) (1.5 g) was dissolved in N-methylpyrrolidone (NMP; $60 \mathrm{ml}$ ) by stirring at $80{ }^{\circ} \mathrm{C}$ for $1 \mathrm{~h}$. Once the polymer had completely dissolved, the solution was heated to $50{ }^{\circ} \mathrm{C}$. A solution of 1-dodecylisocyanate $(0.5 \mathrm{mmol}, 10 \%$ dodecyl modification by weight) was dissolved in NMP $(5 \mathrm{ml})$ and added to the reaction mixture followed by $150 \mu \mathrm{L}$ of $\mathrm{N}, \mathrm{N}$,-diisopropylethylamine as a catalyst. The solution was then stirred at room temperature for $16 \mathrm{~h}$. This solution was then precipitated from acetone and the HPMC- $\mathrm{C}_{12}$ polymer was recovered by filtration, dialyzed within a $3.5 \mathrm{kDa}$ cut-off dialysis bag for 3 days in water, and lyophilized, yielding a white amorphous material. HPMC was found to have a molecular weight of $\mathrm{Mn}(\mathrm{\Xi})=272900$ (1.37) by aqueous size exclusion chromatography (SEC) (full GPC trace shown in Supplementary Figure 2). Aqueous SEC-RI traces were obtained on a Optilab rEX refractive index detector (Wyatt) after passing through a column (Superose 6 Increase 10/300 GL column, Mw range of $5000-5000000 \mathrm{~g} / \mathrm{mol}$ (GE healthcare)). ${ }^{1} \mathrm{H}-\mathrm{NMR}$ was used to confirm modification (Supplementary Figure 3). ${ }^{1} \mathrm{H}-\mathrm{NMR}$ spectra were obtained and recorded on a Varian $600 \mathrm{MHz}$ NMR spectrometer at $298 \mathrm{~K}$, and chemical shifts are given in parts per million. ${ }^{1} \mathrm{H}$-NMR spectra were referenced to residual proton resonances in the deuterated solvents $\left(\right.$ DMSO- $d_{6}$ shift $\left.=2.50\right)$.

\section{PEG-PLA Nanoprecipitation}

Procedure was followed and analyzed as described previously. ${ }^{5}$ A solution (1 mL) of PEG-PLA in acetonitrile $(50 \mathrm{mg} / \mathrm{ml})$ was added dropwise to water $(10 \mathrm{~mL})$ at a stir rate of $600 \mathrm{rpm}$. NPs were purified by ultracentrifugation over a filter (molecular weight cut-off of $10 \mathrm{kDa}$; Millipore Amicon Ultra-15) followed by resuspension in water to a final concentration of $250 \mathrm{mg} / \mathrm{ml}$. NP size and dispersity were characterized by DLS $\left(D_{\mathrm{H}}=35 \mathrm{~nm}, \mathrm{PDI}=0.02\right)$.

\section{PNP Hydrogel Formulation}

HPMC- $\mathrm{C}_{12}$ was dissolved in phosphate-buffered saline at $6 \mathrm{wt} \%$ and loaded into a $1 \mathrm{~mL}$ eppendorf tube. A $20 \mathrm{wt} \%$ PEG-PLA nanoparticle solution in PBS was then added to phosphate buffered saline and loaded into the tube. The contents were thoroughly mixed using a long spatula until gelation occurred. The tube was then spun at $10,000 \mathrm{~g}$ and placed at $4{ }^{\circ} \mathrm{C}$ overnight prior to testing.

\section{Shear Rheology}

Rheological testing was performed using a $20 \mathrm{~mm}$ diameter serrated parallel plate at a $600 \mu \mathrm{m}$ gap on a stresscontrolled TA Instruments DHR-2 rheometer with a solvent trap to prevent dehydration. For lower weight percent formulations, a $40 \mathrm{~mm}$ plate was utilized. All experiments were performed at $25^{\circ} \mathrm{C}$. Frequency sweeps were performed at a strain of $1 \%$. Amplitude sweeps were performed at frequency of $10 \mathrm{rad} / \mathrm{s}$. Flow sweeps were 
performed from high to low shear rates with steady state sensing. Duplicates for nearly all samples were performed for each test, and representative data are presented. G' and $\tan (\delta)$ values are reported at $10 \mathrm{rad} / \mathrm{s}, 1 \%$ strain.

\section{Yield Stress Analysis}

The yield stress was determined by first analyzing the viscosity versus stress from the flow sweep. A tangent line analysis as shown in Figure 6 was used to determine the point of yielding. Next the Herschel-Bulkley equation was fit to the stress versus shear rate data within the flow regime in Prism Software. The Herschel-Bulkley equation,

$$
\sigma=\sigma_{y}+K \dot{\gamma}^{n}
$$

where $\sigma$ is the stress data, $\sigma_{y} K$ is the consistency index, $\dot{\gamma}$ is the associated shear rate, and $n$ is the flow index was fit to determine $\sigma_{y}, K$ and $n$. All fits reported demonstrated $R^{2}$ values above 0.9 .

\section{Viscometry at High Shear Rates}

A Rheosense $m$-VROC viscometer was used to measure the hydrogel viscosity at high shear rates from low to high using a $1 \mathrm{~mL}$ Hamilton syringe. Each data point was collected at steady state.

\section{FRAP Studies and Analysis}

Fluorescein isothiocyanate was coupled to HPMC-C 12 according to literature protocols ${ }^{4,24}$, and included as $\frac{1}{3}$ the wt\% of total polymer added to each hydrogel. Alexa647 was coupled to the PEG-PLA nanoparticles using copper-free click chemistry according to previously published protocols ${ }^{36}$, and included as $\frac{1}{2}$ the wt $\%$ of total nanoparticles added to each hydrogel. Gels were sealed between a glass slide and a coverslip with a $1 \mathrm{~mm}$ gap using an epoxy adhesive and imaged using a confocal LSM780 microscope. Samples were imaged using a low intensity laser to observe an initial level of fluorescence. Then the laser was switched to full intensity and focused on a region of interest $(\mathrm{ROI})$ with a $25 \mu \mathrm{m}$ diameter for 10 seconds in order to bleach a circular area. If the 10 seconds of bleaching did not reduce the fluorescence in the ROI by more than $15 \%$, an additional bleach was applied. Fluorescence data were then recorded until a plateau was observed to create an exponential fluorescence recovery curve. Samples were taken from different regions of each gel $(n=3-4)$. The diffusion coefficient was calculated according to,

$$
D=\frac{\gamma_{D} \omega^{2}}{4 \tau_{1 / 2}}
$$

where the constant $D=\frac{\tau_{1 / 2}}{\tau_{D}}$ with $\tau_{1 / 2}$ being the half-time of the recovery, $\tau_{D}$ the characteristic diffusion time, both yielded by the ZEN software, and $\omega$ the radius of the bleached ROI $(12.5 \mu \mathrm{m})^{24}$.

\section{Interparticle Spacing Calculation}

The interparticle spacing (IPS) was calculated by assuming close random packing and the following equation ${ }^{37}$ :

$$
I P S=2 r\left(\left(\sigma_{m} / \sigma\right)^{1 / 3}-1\right)
$$

where $\sigma_{m}$ is $0.63, \sigma$ is the particle volume fraction, and $r$ is the radius of the particle.

\section{Statistical Analysis}

All error is reported as standard deviation unless otherwise specified. P values were calculated from Student's unpaired $t$ tests.

\section{Competing interests}

The authors declare that they have no competing interests.

\section{Data Availability}

The data that support the findings of this study are available upon reasonable request from the corresponding author. The data are not publicly available due to privacy or ethical restrictions. 


\section{Author's contributions}

A.K.G. and E.A.A. conceived of the project and performed experiments. O.A.S. assisted with diffusion experiments and analysis. H.L. aided in data interpretation and experimental planning. All authors contributed to the manuscript.

\section{Acknowledgements}

This research was financially supported by the Center for Human Systems Immunology with the Bill and Melinda Gates Foundation (OPP1113682; OPP1211043), the Stanford Bio-X Interdisciplinary Initiatives Program Seed Grant program, the American Cancer Society (RSG-18-133-01-CDD), and the Goldman Sachs Foundation (administered by the Stanford Cancer Institute, SPO 162509). A.K.G.is thankful for a National Science Foundation Graduate Research Fellowship and the Gabilan Fellowship of the Stanford Graduate Fellowship in Science and Engineering. H.L.H. was also partially supported by the NSF AGEP postdoctoral fellowship. Authors would also like to acknowledge Anthony Yu for helpful discussions.

\section{References}

1. Correa, S. et al. Translational applications of hydrogels. Chemical Reviews (2020).

2. Anthony, C. Y. et al. Physical networks from entropy-driven non-covalent interactions. Nature communications 12, 1-9 (2021).

3. Appel, E. A. et al. Self-assembled hydrogels utilizing polymer-nanoparticle interactions. Nature communications 6, 1-9 (2015).

4. Roth, G. A. et al. Injectable hydrogels for sustained codelivery of subunit vaccines enhance humoral immunity. ACS Central Science 6, 1800-1812, DOI: 10.1021/acscentsci.0c00732 (2020). https://doi.org/10.1021/acscentsci. 0c00732.

5. Grosskopf, A. K. et al. Injectable supramolecular polymer-nanoparticle hydrogels enhance human mesenchymal stem cell delivery. Bioengineering \& Translational Medicine 5, e10147 (2020).

6. Fenton, O. S. et al. Injectable polymer-nanoparticle hydrogels for local immune cell recruitment. Biomacromolecules 20, 4430-4436 (2019).

7. Meis, C. M. et al. Self-assembled, dilution-responsive hydrogels for enhanced thermal stability of insulin biopharmaceuticals. ACS Biomaterials Science \& Engineering (2020).

8. Stapleton, L. M. et al. Use of a supramolecular polymeric hydrogel as an effective post-operative pericardial adhesion barrier. Nature Biomedical Engineering 3, 611-620 (2019).

9. Anthony, C. Y. et al. Wildfire prevention through prophylactic treatment of high-risk landscapes using viscoelastic retardant fluids. Proceedings National Academy Sciences 116, 20820-20827 (2019).

10. Guzzi, E. A., Bovone, G. \& Tibbitt, M. W. Universal nanocarrier ink platform for biomaterials additive manufacturing. Small 15, 1905421 (2019).

11. Anthony, C. Y. et al. Scalable manufacturing of biomimetic moldable hydrogels for industrial applications. Proceedings National Academy Sciences 113, 14255-14260 (2016).

12. Lopez Hernandez, H., Grosskopf, A. K., Stapleton, L. M., Agmon, G. \& Appel, E. A. Non-newtonian polymernanoparticle hydrogels enhance cell viability during injection. Macromolecular bioscience 19, 1800275 (2019).

13. Meis, C. M., Grosskopf, A. K., Correa, S. \& Appel, E. A. Injectable supramolecular polymer-nanoparticle hydrogels for cell and drug delivery applications. J. Vis. Exp 168, e62234 (2021).

14. Lopez Hernandez, H., Souza, J. W. \& Appel, E. A. A quantitative description for designing the extrudability of shear-thinning physical hydrogels. Macromolecular Bioscience 2000295 (2020).

15. Gu, L. \& Mooney, D. J. Biomaterials and emerging anticancer therapeutics: engineering the microenvironment. Nature Reviews Cancer 16, 56 (2016).

16. Axpe, E. et al. A multiscale model for solute diffusion in hydrogels. Macromolecules 52, 6889-6897 (2019).

17. Richbourg, N. R. \& Peppas, N. A. The swollen polymer network hypothesis: Quantitative models of hydrogel swelling, stiffness, and solute transport. Progress Polymer Science 105, 101243 (2020). 
18. Anthony, C. Y., Smith, A. A. \& Appel, E. A. Structural considerations for physical hydrogels based on polymernanoparticle interactions. Molecular Systems Design \& Engineering 5, 401-407 (2020).

19. Agrawal, A., Yu, H.-Y., Sagar, A., Choudhury, S. \& Archer, L. A. Molecular origins of temperature-induced jamming in self-suspended hairy nanoparticles. Macromolecules 49, 8738-8747 (2016).

20. Agarwal, P., Qi, H. \& Archer, L. A. The ages in a self-suspended nanoparticle liquid. Nano letters 10, 111-115 (2010).

21. Pagels, R. F. \& Prud'Homme, R. K. Polymeric nanoparticles and microparticles for the delivery of peptides, biologics, and soluble therapeutics. Journal Controlled Release 219, 519-535 (2015).

22. Danafar, H., Rostamizadeh, K., Davaran, S. \& Hamidi, M. Drug-conjugated pla-peg-pla copolymers: a novel approach for controlled delivery of hydrophilic drugs by micelle formation. Pharmaceutical development technology 22, 947-957 (2017).

23. Mašková, E. et al. Hypromellose-a traditional pharmaceutical excipient with modern applications in oral and oromucosal drug delivery. Journal Controlled Release 324, 695-727 (2020).

24. Axelrod, D., Koppel, D., Schlessinger, J., Elson, E. \& Webb, W. W. Mobility measurement by analysis of fluorescence photobleaching recovery kinetics. Biophysical journal 16, 1055-1069 (1976).

25. Cai, P. C. et al. Dynamic light scattering microrheology for soft and living materials. Soft Matter 17, 1929-1939 (2021).

26. Rolere, S., Cartault, M., Sainte-Beuve, J. \& Bonfils, F. A rheological method exploiting cole-cole plot allows gel quantification in natural rubber. Polymer Testing 61, 378-385 (2017).

27. Brassinne, J., Cadix, A., Wilson, J. \& Van Ruymbeke, E. Dissociating sticker dynamics from chain relaxation in supramolecular polymer networks - the importance of free partner! Journal Rheology 61, 1123-1134 (2017).

28. Aguado, B. A., Mulyasasmita, W., Su, J., Lampe, K. J. \& Heilshorn, S. C. Improving viability of stem cells during syringe needle flow through the design of hydrogel cell carriers. Tissue Engineering Part A 18, 806-815 (2012).

29. Donley, G. J., Singh, P. K., Shetty, A. \& Rogers, S. A. Elucidating the g overshoot in soft materials with a yield transition via a time-resolved experimental strain decomposition. Proceedings National Academy Sciences 117, 21945-21952 (2020).

30. Hartnett, J. P. \& Hu, R. Y. The yield stress—an engineering reality. Journal Rheology 33, 671-679 (1989).

31. Ewoldt, R. H., Johnston, M. T. \& Caretta, L. M. Experimental challenges of shear rheology: how to avoid bad data. In Complex fluids in biological systems, 207-241 (Springer, 2015).

32. Burgos, G. R., Alexandrou, A. N. \& Entov, V. On the determination of yield surfaces in herschel-bulkley fluids. Journal Rheology 43, 463-483 (1999).

33. Grosskopf, A. K. et al. Viscoplastic matrix materials for embedded 3d printing. ACS applied materials \& interfaces 10, 23353-23361 (2018).

34. Nelson, A. Z. \& Ewoldt, R. H. Design of yield-stress fluids: A rheology-to-structure inverse problem. Soft matter 13, 7578-7594 (2017).

35. Denn, M. M. \& Bonn, D. Issues in the flow of yield-stress liquids. Rheologica acta 50, 307-315 (2011).

36. Saouaf, O. M. et al. Modulation of injectable hydrogel properties for slow co-delivery of influenza subunit vaccine components enhance the potency of humoral immunity. Journal Biomedical Materials Research Part A (2021).

37. Hao, T. \& Riman, R. E. Calculation of interparticle spacing in colloidal systems. Journal colloid interface science 297, 374-377 (2006). 